\title{
CASE REPORT: Diagnosing Thoracic Aortic Dissection using Bedside Ultrasound in an Emergency Department in a Norwegian University Teaching Hospital
}

Jay Chotai M.D. ${ }^{1}$,

Tone Dolva Dahl M.D., Ph.D. ${ }^{2}$, Irida Vevecka, M.D. ${ }^{3}$

${ }^{1}$ Department of Emergency Medicine, Department of Haematology, Akershus University Hospital

2 Department of Internal Medicine, Akershus University Hospital

${ }^{3}$ Department of Cardiology, Akershus University Hospital

\section{Abstract}

Thoracic aortic dissection (TAD) is a medical emergency which can present with a variety of symptoms. It is vital to rapidly identify and diagnose this condition to increase the patients' chances of survival. Computed tomography (CT) and Magnetic resonant imaging (MRI) are the standard diagnostic modalities, however these are normally carried out outside an emergency department (ED), which could delay diagnosis and delivery of care.

Transthoracic Echocardiography (TTE) is an accepted first line imaging modality in the ED. This has been previously reported by the European Association of Echocardiography [1], and ultrasound is becoming widely available and used in EDs nowadays. The aim of this article is to present a case in which bedside ultrasound with focused cardiac examination (FOCUS) was utilised to provide a rapid diagnosis of a clinical suspected aortic dissection.

\section{Case}

This case reports a 55-year-old male who was admitted to the ED at Akershus Universi- ty Teaching Hospital, Norway. The symptoms included chest pain radiating to the left jaw, with concomitant visual disturbances to his left eye. He was a former smoker and had a history of asthma and borderline hypertension. On admission, the patient had no complaints except for mild pain in his left jaw and chest as described previously, with now a normalised vision. The physical examination showed a significant blood pressure difference in the upper extremities; $135 / 85 \mathrm{mmHg}$ on left arm and $100 / 70 \mathrm{mmHg}$ on right arm. Other vital signs were unremarkable. He had a strong holosystolic cardiac murmur, which was not previously described. There were no palpable masses in the abdomen, and the neurological examination was normal. ECG was without signs of acute ischemia. His lab test showed haemoglobin of $15.4 \mathrm{~g} / \mathrm{dL}$, CRP $3 \mathrm{mg} / \mathrm{L}$, ALAT $65 \mathrm{U} / \mathrm{L}$, creatinine $104 \mu \mathrm{mol} / \mathrm{L}$, troponin T $9 \mathrm{ng} / \mathrm{L}$, NT-proBNP $67 \mathrm{ng} / \mathrm{L}$ and lactate $3.1 \mathrm{mmol} / \mathrm{L}$.

Bedside ultrasound with FOCUS revealed a dilated aortic root of $53 \mathrm{~mm}$ ( $\vee$ Fig. 1) and a proximal ascending aorta of $43 \mathrm{~mm}$ ( $\triangleright$ Fig.3). The FOCUS parasternal long axis projection and suprasternal projection, showed an intimal flap in the aortic arch and ascending aorta extending down to the aortic valve. This can be visualised in - Fig. 2, 4, 5 below. There was a moderate aortic regurgitation ( $>$ Fig. 5 ), which was presumed secondary to the aortic dissection. There was no pericardial effusion, and the abdominal aorta was not examined.

A CT was requested and confirmed a Stanford type $A$ aortic dissection from the aortic valve to below the renal arteries. The patient was transferred to the thoracic sur-

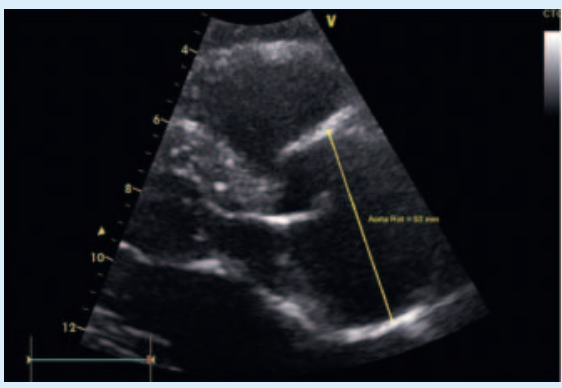

- Fig. 1 2D Parasternal long axis view, visualisation of a dilated aortic root $53 \mathrm{~mm}$ (yellow line).

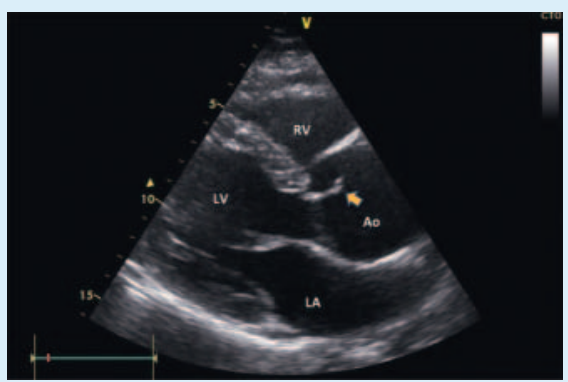

- Fig. 2 2D Parasternal long axis view with visualisation of intimal flap in the aortic root (yellow arrow). Ao: aorta; $R V$ : right ventricle; $L V$ : left ventricle; $L A$ : left atrium.

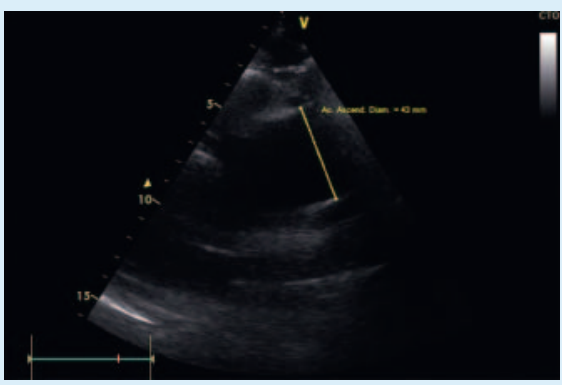

- Fig. 3 2D Parasternal long axis of aorta with visualisation of a dilated aortic ascendens $43 \mathrm{~mm}$ (yellow line). 


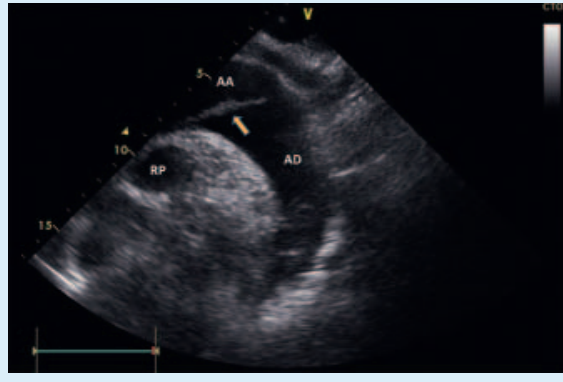

- Fig. 4 2D Suprasternal view of the aorta, with visualisation of intimal flap (yellow arrow) in the aortic arch. AA: aortic arch; $A D$ : aorta descendent; $R P$ : right pulmonary artery.

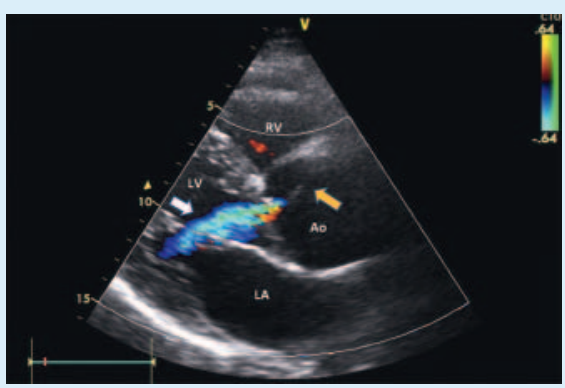

- Fig. 5 2D color doppler mode parasternal long axis, with visualization of a moderate aortic regurgitation (white arrow) and intimal flap (yellow arrow). Ao: aorta; $R V$ : right ventricle; $L V$ : left ventricle; $L A$ : left atrium.

gery facility at Oslo University Hospital and underwent urgent surgery.

\section{Discussion}

Thoracic Aortic Dissection (TAD) is a rare condition, with high mortality if untreated. The estimated incidence is $5-10$ cases per million, and of the most common underlying medical condition is hypertension [2]. The symptoms and signs of TAD can be seen with other critical medical conditions and is very variable in presentation. Chest pain is present in up to $90 \%$ of patients upon admission [3].

Survival rates are increased with early diagnosis, especially when the dissection involves the aortic root which can cause a secondary myocardial infarction or aortic valve failure [4]. The mortality rate is as high as $1 \%$ per hour in the first 48 hours without the provision of the necessary treatment [4]. It is deemed important for clinicians to maintain a high level of suspicion to detect the diagnosis and provide treatment as early as possible. CT and MRI are current standard diagnostic modalities which have largely replaced aortography [5]. Nowadays TTE is widely accessible to emergency physicians, and a focused examination of the heart is a part of ultrasound performance protocols utilised bedside in different settings in emergency departments. Standard imaging of a focus cardiac ultrasound examination (FOCUS) usually includes parasternal long axis, parasternal short axis, and apical view of the heart. The sensitivity and specificity for ultrasound for detection of aortic dissection has been shown to be high in selected studies [6], but the true sensitivity and specificity is probably variable depending on the level of experience of the operator performing the examination [7]. Although two echocardiographic features are strongly suggestive of thoracic aortic dissection; visualization of dilatation of any segment of the aorta, and visualisation of an intimal flap in the aorta [8]. FOCUS in combination with certain clinical risk factor for diagnosing aortic dissection also results in high sensitivity and specificity for diagnosing of Aortic Dissection [9].

\section{Conclusion}

Bedside ultrasound with FOCUS can be performed by emergency physicians as a screening tool in patients with suspected thoracic aortic dissection. The examination can be performed in a very short space of time, rendering it a very efficient modality of diagnosis for well-trained emergency physicians. This will ensure patients' diagnosis efficiently and ensure further aortic imaging or urgent surgical evaluation where indicated. Ultrasonographic sign of an intimal flap of the aorta, and aortic segment dilatation has been shown to be sensitive and specific for the diagnosis of thoracic aortic dissection, which was found in our case. It is important to note that FOCUS cannot be used as a standalone test to rule in or rule out thoracic aortic dissection.

\section{Conflict of Interest}

Employed on an advisory board of Sanofi Genzyme.

Received lecture fees from Pfizer and Bayer AS.

\section{References}

[1] Evangelista A, Flachskampf FA, Erbel R et al. European Association of Echocardiography; Document Reviewers:, Pepi M, Breithardt OA, Plonska-Gosciniak E. Echocardiography in aortic diseases: EAE recommendations for clinical practice. Eur J Echocardiogr 2010; 11: 645658. doi:10.1093/ejechocard/jeq056. Erratum in: Eur J Echocardiogr. 2011 Aug;12(8):642. PMID: 20823280.

[2] Sullivan PR, Wolfson AB, Leckey RD et al. Diagnosis of acute thoracic aortic dissection in the emergency department. The American Journal of Emergency Medicine 2000; 18: 46-50, ISSN 0735-6757, https://doi.org/10.1016/ S0735-6757(00)90047-0.

[3] Strayer R], Shearer PL, Hermann LK. Screening, evaluation, and early management of acute aortic dissection in the ED. Curr Cardiol Rev 2012; 8: 152-157. doi:10.2174/157340312801784970. PMID: 22708909; PMCID: PMC3406274.

[4] Blaivas M, Sierzenski PR. Dissection of the proximal thoracic aorta: a new ultrasonographic sign in the subxiphoid view. Am J Emerg Med 2002; 20: 344-348. doi:10.1053/ ajem.2002.33006. PMID: 12098184.

[5] Williams ], Heiner JD, Perreault MD et al. Aortic dissection diagnosed by ultrasound. West J Emerg Med 2010; 11: 98-99

[6] Victor MF, Mintz GS, Kotler MN et al. Two dimensional echocardiographic diagnosis of aortic dissection. Am J Cardiol 1981; 48: 1155

[7] Fojtik JP, Costantino TG, Dean AJ. The diagnosis of aortic dissection by emergency medicine ultrasound. J Emerg Med 2007; 32: 191-196. doi:10.1016/j.jemermed.2006.07.020. Epub 2007 Jan 22. PMID: 17307632.

[8] Roudaut RP, Billes MA, Gosse P et al. Accuracy of M-mode and two-dimensional echocardiography in the diagnosis ofaortic dissection: An experience with 128 cases. Clin Cardiol 1988; 11: 553-562

[9] Nazerian P, Vanni S, Castelli M et al. Diagnostic performance of emergency transthoracic focus cardiac ultrasound in suspected acute type A aortic dissection. Intern Emerg Med 2014; 9: 665-670. doi:10.1007/s11739-014-1080-9. Epub 2014 May 29. PMID: 24871637. 